\title{
DIE KOSTENMINIMALE BELASTUNG OFFENER WARTESYSTEME MIT POISSON-INPUT DER FORDERUNGEN
}

\section{EINLEITUNG}

Im folgenden werden offene Bedienungssysteme mit strengem Wartecharakter und Poisson-Ankunft der Forderungen untersucht. Im einzelnen werden Methoden zur Bestimmung der kostenminimalen Belastung eines solchen Bedienungssystems erörtert.

Dabei ist unter einer kostenminimalen Belastung eines Bedienungssystems eine solche Ankunftsintensität der Forderungen zu verstehen, für die die mittleren Kosten, die das Bedienungssystem in der Zeiteinheit verursacht, minimal ausfallen. Die Ermittlung der kostenminimalen Belastung ist vor allem für die Intensivierung bereits vorhandener Wartesysteme (insbesondere von Umschlagssystemen) von praktischer Bedeutung.

\section{WARTE SYSTEME DER FORM $M / M / s(s \geq 2)$}

2.1. DIE KOSTENFUNKTION

Zunächst werden offene Wartesysteme mit Poisson-Input der Forderungen und mit einer exponentiell verteilten Abfertigungszeit betrachtet. Die Kostenfunktion, die die mittleren Kosten eines offenen Bedienungssystems in einer Zeiteinheit darstellt, lautet nunmehr [3]

$$
\boldsymbol{K}=(s-\varrho) k_{f}+\varrho k_{b}+L_{w} k_{w}
$$

mit $\varrho=\lambda / \mu$ als Verkehrsdichte und

$$
L_{w}=\frac{\varrho^{s+1}}{(s-\varrho)\left[(s-\varrho)(s-1) ! \sum_{r=0}^{s-1}\left(\varrho^{r} / r !\right)+\varrho^{s}\right]}
$$


als mittlere Schlangenlänge des Bedienungssystems. Weiter bedeuten $s$ - die Anzahl der (äquivalenten, absolut zuverlässigen und parallel angeordneten) Bedienungsstellen, $\lambda-$ die Ankunftsintensität der Forderungen und $\mu$-die Abfertigungsintensität einer Bedienungsstelle sowie $k_{f}-$ die mittleren Kosten einer freien Bedienungsstelle, $k_{b}-$ die mittleren Kosten einer besetzten Bedienungsstelle und $k_{w}$ - die mittleren Kosten einer wartenden Forderung (jeweils bezogen auf eine bestimmte Zeiteinheit). Selbstverständlich wird $\varrho<s$ oder

$$
\lambda<s \mu
$$

angenommen, da andernfalls das betrachtete Wartesystem kritisch ist und damit sein Kostenverhalten gegenstandslos ist.

Es ist unmittelbar evident, daß die mittleren Kosten $K$ eine konvexe Funktion des stetigen Parameters $\lambda$ im Intervall $0 \leqslant \lambda<s \mu$ sind. Mithin gibt es genau einen Wert $\lambda=\lambda^{*}$, der den Ausdruck (1) - bei Festhaltung der übrigen unabhängigen Größen - minimiert. Dieser Wert wird im folgenden die kostenminimale Belastung des Wartesystems genannt. Es gibt verschiedene Verfahren zur Bestimmung dieser Größe $\lambda^{*}$, auf die nun eingegangen wird.

\subsection{VERFAHREN ZUR BESTIMMUNG VON $\lambda^{*}$}

2.2.1. Tabellierung. Die Funktion (1) wird im Intervall $0 \leqslant \lambda<s \mu$ tabelliert. Dabei ist es zweckmäßig, die Kostenfunktion $K$ auf Ausdrücke zurückzuführen, die bereits in Tafeln vorliegen. Es gilt

$$
L_{w}=\frac{\varrho^{2}}{(s-\varrho)[\varrho+(s-\varrho) R(\varrho, s-1) / P(\varrho, s)]}
$$

mit

$$
P(a, j)=\frac{a^{j} e^{-a}}{j !}, \quad R(a, j)=\sum_{i=0}^{j} P(a, i) .
$$

Die Poisson-Funktionen (4) sind in verschiedenen Büchern der Wahrscheinlichkeitsrechnung (z. B. in [1]) tabelliert. Diese Methode ist relativ aufwendig und umständlich. Weniger unbequem ist eine Tabellierung der Kostenfunktion $K$ bei Zuhilfenahme von Tafeln für die mittlere Schlangenlänge $L_{w}$. Solche Tafeln sind beispielsweise in [3] für $0<\varrho<s$ und $s=1(1) 20$ angegeben. Aber auch diese Vorgehensweise ist mühsam, insbesondere für Stabilitätsbetrachtungen hinsichtlich der kostenminimalen Belastung $\lambda^{*}$.

2.2.2. Diagramme. Vorteilhafter als die Anwendung der bisher skizzierten Verfahren ist die Benutzung von Diagrammen, die nicht 
nur eine schnelle Auffindung des Wertes $\lambda=\lambda^{*}$ ermöglichen, sondern auch ohne nennenswerten Aufwand Empfindlichkeitsbetrachtungen zulassen. Solche Diagramme sind bisher von Gorbatij [2] und Rossa [4] vorgeschlagen worden. Im folgenden werden diese Diagramme charakterisiert und einige ihnen anhaftende Mängel behoben.

2.2.2.1. Das Diagramm von Gorbatij. Gorbatij geht davon aus, daß die Kostenfunktion $K$ eines offenen Wartesystems eine konvexe Funktion in $\varrho$ ist. Demzufolge gibt es wenigstens einen Wert $\varrho=\varrho^{*}$, der die Kostenfunktion minimiert. Diese kostenminimale Verkehrsdichte $\varrho^{*}$ ist offenbar die einzige Nullstelle der algebraischen Gleichung

$$
\frac{\partial K}{\partial \varrho}=0
$$

Die kostenminimale Belastung $\lambda^{*}$ ergibt sich bei bekannter kostenminimaler Verkehrsdichte $\varrho^{*} \mathrm{zu}$

$$
\lambda^{*}=\varrho^{*} \mu \text {. }
$$

Gorbatij bestimmt nun für verschiedene Werte der Dimension $s$ und des Kostenverhältnisses $c=k_{f} / k_{w}$ die kostenminimale Verkehrsdichte (als einzige Nullstelle der Gleichung (5)) und entwickelt daraus ein Diagramm für die Bestimmung von $\varrho^{*}$ und zwar für den Bereich $s=2(1) \mathbf{1 0}$ und $0 \leqslant c \leqslant 4$. Entsprechend (6) kann dieses Diagramm zur Ermittlung der kostenminimalen Belastung $\lambda^{*}$ benutzt werden.

Der erste Fehler der Untersuchungen von Gorbatij besteht darin, daß in der Kostenfunktion (1) die von $\lambda$ abhängigen mittleren Abfertigungskosten $\varrho k_{b}$ vernachlässigt werden. Dieser Fehler kann aber dadurch ausgeglichen werden, daß im Diagramm das Kostenverhältnis $c=k_{f} / k_{w}$ durch den Kostenquotienten $c^{\prime}=\left(k_{f}-k_{b}\right) / k_{w}$ ersetzt wird. Der zweite Mangel liegt darin, daß die Bestimmungsgleichung (5) - abgesehen von der bereits erwähnten Vernachlässigung der mittleren Abfertigungskosten - unvollständig angegeben worden ist (möglicherweise liegt ein Druckfehler vor). Sie muß bei Berücksichtigung der mittleren Abfertigungskosten und mit den von uns eingeführten Bezeichnụngen richtig folgendermaßen lauten:

mit

$$
\begin{array}{r}
\frac{k_{f}-k_{b}}{k_{w}}=\frac{\varrho^{s}}{N}\left[s+1-\frac{\varrho}{N}\left\{( s - 1 ) ! \left(2(\varrho-s) \sum_{k=0}^{s-1} \frac{\varrho^{k}}{k !}\right.\right.\right. \\
\left.+(s-\varrho)^{2} \sum_{k=0}^{s-2} \frac{\varrho^{k}}{k !}\right)+ \\
\left.\left.+\varrho^{s-1}\left(s^{2}-s \varrho-\varrho\right)\right\}\right]
\end{array}
$$

$$
N=(s-\varrho)^{2}(s-1) ! \sum_{k=0}^{s-1} \frac{\varrho^{k}}{k !}+\varrho^{s}(s-\varrho)
$$


2.2.2.2. Das Diagramm von Rossa. Rossa geht in seinen Überlegungen ähnlich wie Gorbatij vor. Er betrachtet noch mögliche Erlöse, die bei der Abfertigung für den Betreiber eines Wartesystems entstehen. Da die Erlöse aber in den mittleren Abfertigungskosten (Erlöse als negative Kosten) berücksichtigt werden können, unterscheidet sich die Kostenfunktion von Rossa nicht von dem Ausdruck (2). Die Kostenparameter sind allerdings bei Rossa unklar definiert; es fehlt ihre Zeitbezogenheit. Außerdem ist seine Gewinnfunktion (möglicherweise infolge Druckfehler) falsch angegeben. Sie muß richtig lauten

$$
J=a \lambda-\left[\varrho k_{b}+(s-\varrho) k_{f}+L_{w} k_{w}\right]
$$

mit $a$ als Erlös, der bei der Abfertigung einer Forderung erzielt wird. Bei der Ableitung dieser Funktion nach dem Parameter $\varrho$ unterschlägt Rossa den Erlösanteil $a \lambda$, der offensichtlich von $\varrho$ abhängt. Daher sind die anschließenden Darlegungen von Rossa nur bedingt richtig. Sie lassen sich aber folgendermaßen retten. Wegen $a \lambda=\varrho g_{b}$ mit $g_{b}$ als Erlös, der während einer eine Zeiteinheit dauernde Abfertigung entsteht, braucht nur die spezifische Kostengröße $k_{b}$ durch den Parameter $k_{b}^{\prime}=k_{b}-g_{b}$ ersetzt zu werden. Treten keine Erlöse auf, dann sind die Überlegungen von Rossa wegen $a=0$ oder $g_{b}=0$ gültig.

Rossa berechnet für verschiedene Werte des Quotienten $c^{\prime}$ und der Dimension $s$ auf der Grundlage der (5) entsprechenden Beziehung die kostenminimale Verkehrsdichte $\varrho^{*}$. Daraus leitet er ein Diagramm ab, das eine Kurvenschar $\varrho_{s}^{*}\left(c^{\prime}\right)$ darstellt. Dieses Diagramm eignet sich für eine bequeme Bestimmung von $\varrho^{*}$ und damit auch von $\lambda^{*}$. Dieses Diagramm, das sehr grob ist und dessen Eingangsgrößen unzureichend präzisiert sind, ist allerdings weniger brauchbar für die kostenminimale (oder gewinnmaximale) Dimensionierung eines Wartesystems, $d$. $h$. für die Bestimmung einer kostenminimalen Anzahl von Bedienungsstellen, da es keine Stabilitätsbereiche für die kostenminimale Dimension unmittelbar angibt. Rossa überschätzt die Anwendungsmöglichkeiten seines Diagramms auch insofern, als er es für die Bestimmung einer kostenminimalen Abfertigungsrate geeignet hält. Das ist aber ausgeschlossen. Die spezifischen Kostenparameter $k_{b}$ und $k_{f}$ (Eingangsgrößen des Diagramms) sind nämlich selbst Funktionen der Abfertigungsrate $\mu$, deren Struktur weitgehend noch ungeklärt ist. Es ist nicht einmal bekannt unter welchen Bedingungen eine kostenminimale Bedienungsrate für ein Wartesystem der Form $M / M / s$ überhaupt existiert. Lediglich für einstellige Wartesysteme der Gestalt $M / G / 1$ lassen sich entsprechende Existenz- und Unitätsaussagen machen.

2.2.3. Diagramme und Tabellierung. Abschließend wird ein Verfahren zur Bestimmung von $\lambda^{*}$ erörtert, das eine Kombination aus der Anwendung eines Diagramms und einer Tabellierung der Kostenfunktion (1) 
beinhaltet. Es handelt sich hierbei um ein Diagramm zur kostenminimalen Dimensionierung eines offenen Wartesystems [5], das in [3] ausführlich begründet und dargestellt ist. Die Abb. 1 gibt einen Ausschnitt aus diesem umfangreichen Diagramm, das unmittelbar die zu dem Kosten-

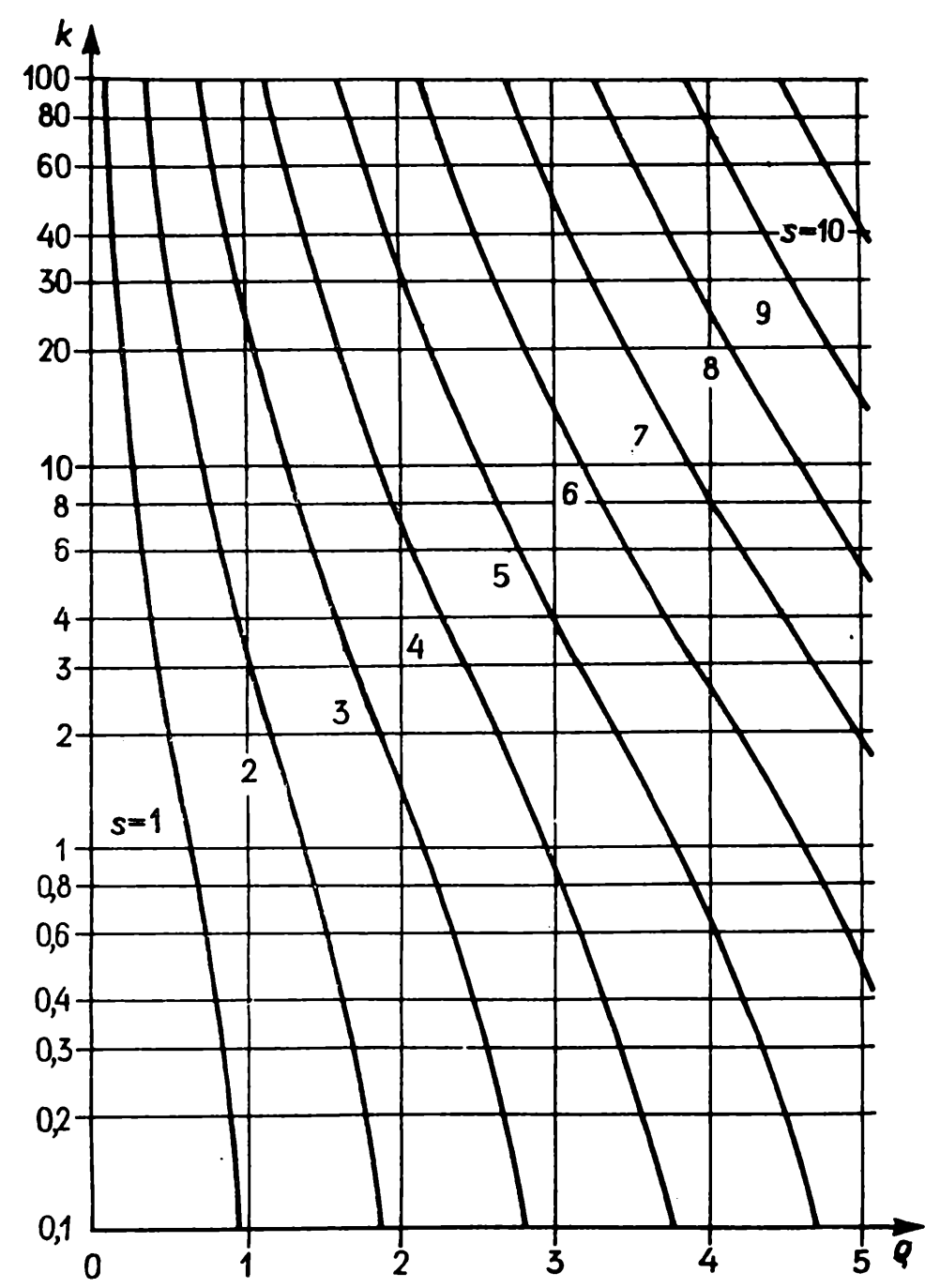

Abb. 1. Diagram zur kostenminimalen Dimensionierung eines offenen Wartesystems der Gestalt $M / M / s$

verhältnis $k=k_{w} / k_{f}=1 / c$ und zu dem Verkehrswert $\varrho$ gehörige kostenminimale Dimension $s^{*}$ sowie deren Stabilitätsbereich ausweist. Sind nun die Werte $k$ und $s$ gegeben und ist die kostenminimale Belastung gesucht, dann wird zunächst mit Hilfe des Diagramms der $\varrho$-Bereich ermittelt, für den die gegebene Dimension $s$ kostenminimal ist. Innerhalb dieses Bereiches wird sodann die kostenminimale Verkehrsdichte $e^{*}$ durch eine Tabellierung von (1) gesucht. Sind beispielsweise die Werte $\mu=1 / 6$, $s=5, k_{b}=-4, k_{f}=3$ und $k_{w}=5$ vorgegeben, dann ergibt sich gemä $B$ der Abb. $1 \mathrm{zu}$ dem Kostenverhältnis $k=5 / 3$ das Intervall $2,7 \leqslant \varrho \leqslant 3,5$, 
für das $s=5$ Bedienungsstellen ein Kostenminimum bewirken. Eine Tabellierung der Kostenfunktion $\boldsymbol{K}$ liefert die kostenminimale Verkehrsdichte $\varrho^{*}=3,4, \mathrm{zu}$ der infolge $\mu=1 / 6$ und Relation (6) die kostenminimale Belastung $\lambda^{*}=\mathbf{0 , 5 7}$ gehört.

Die kostenminimale Verkehrsdichte $\varrho^{*}$ muß aber nicht notwendigerweise dem $\varrho$-Intervall angehören, das bei vorgegebenen Werten für $k$ und $s$ mittels des Diagramms konstatiert wird. Das liegt daran, daß das zur Diskussion stehende Diagramm nur auf dem von $s$ abhängigen Bestandteil

$$
C(s)=s+k L_{w}(\varrho, s)
$$

der Kostenfunktion (1) beruht. Wegen

$$
K=\varrho\left(k_{b}-k_{f}\right)+k_{f} C(s)
$$

tritt dieser Fall (des Nichtvorhandenseins von $\varrho^{*}$ im betreffenden $\varrho$-Bereich) insbesondere dann auf, wenn der Term $\left|\varrho\left(k_{b}-k_{f}\right)\right|$ sehr groß ist oder die Freikosten $k_{f}$ erheblich von 1 abweichen. In einem solchen Falle muß die Tabellierung der mittleren Kosten $K$ über das $\varrho$-Intervall hinaus fortgesetzt werden. Welche Seite des Intervalls dabei zu überschreiten ist, geht aus der Tendenz der bereits innerhalb des Intervalls berechneten Werte der Kostenfunktion hervor.

\section{WARTESYSTEME DER FORM $M / G / s \quad(s \geqslant 2)$}

Es wird nun die Voraussetzung fallen gelassen, daß die Zeit für die Bedienung einer Forderung einer (negativen) Exponentialverteilung genügt. Alle übrigen Voraussetzungen (und Bezeichnungen) des Abschnitts 2 werden beibehalten. Einer Bestimmung der kostenminimalen Belastung $\lambda^{*}$ sind nun erhebliche Schranken gesetzt. Es können nunmehr nur Näherungswerte bestimmt werden, über deren Genauigkeit noch nichts ausgesagt werden kann. Lediglich der Fall

$$
k_{b} \geqslant k_{f}
$$

läßt sich exakt behandeln. Unter der Bedingung (7) verlangt eine tätige Bedienungsstelle nicht weniger Aufwand als eine wartende Abfertigungsstelle. Das ist sicher dann der Fall, wenn die Bedienung der Forderungen keinen Erlös für den Betreiber der Bedienungsstellen abwirft. Daher entstehen nunmehr die geringsten (mittleren) Kosten, wenn überhaupt keine Forderung dem Wartesystem zugeführit wird, d. h. wenn $\lambda^{*}=0$ gilt. Dieser Sonderfall ist aber für die Praxis kaum von Interesse.

Die mittleren Kosten $K$, die ein Wartesystem der Gestalt $M / G / s$ (mit $s \geqslant 2$ ) in der Zeiteinheit verursacht, lassen sich durch den Näherungsausdruck [3] 


$$
K=(s-\varrho) k_{f}+\varrho k_{b}+\frac{1+v^{2}}{2} k_{w} L_{w}
$$

erfassen. Dabei bedeutet $v$ den Variationskoeffizient der Abfertigungszeit. Die rechte Seite dieser Näherungsrelation geht offenbar in die Kostenfunktion (1) eines Wartesystems der Form $M / M / s$ über, wenn die spezifischen Wartekosten $k_{w}$ zu

$$
k_{w}^{\prime}=k_{w} \frac{1+v^{2}}{2}
$$

modifiziert werden. Demzufolge lassen sich die unter Punkt 2.2 angegebenen Verfahren für die näherungsweise Ermittlung der kostenminimalen Belastung eines Wartesystems $M / G / s$ anwenden, sobald anstelle des Kostenparameters $k_{w}$ die Größe $k_{w}^{\prime}$ tritt.

\section{WARTESYSTEME DER FORM $M / G / 1$}

Es wird nun ein einstelliges Wartesystem mit Poisson-Input der Forderungen und beliebig verteilter Abfertigungszeit untersucht. Dabei wird der triviale Sonderfall (7) ausgeschlossen. Für die Kostenfunktion dieses Bedienungssystems gilt folgender geschlossener Ausdruck [3]:

$$
K=(1-\varrho) k_{f}+\varrho k_{b}+\frac{\varrho^{2}\left(1+v^{2}\right)}{2(1-\varrho)} k_{w}
$$

Unter der selbstverständlichen Bedingung $0 \leqslant \varrho<1$ (vgl. Relation (3)!) läßt sich für die einzige Nullstelle der Gleichung (5) der analytische Ausdruck

$$
\varrho^{*}=1-\sqrt{\frac{k_{w}\left(1+v^{2}\right)}{2\left(k_{f}-k_{b}\right)+k_{w}\left(1+v^{2}\right)}}
$$

angeben, der die kostenminimale Verkehrsdichte darstellt. Für die kostenminimale Belastung gilt dann entsprechend (6) der Term

$$
\lambda^{*}=\mu\left[1-\sqrt{\frac{k_{w}\left(1+v^{2}\right)}{2\left(k_{f}-k_{b}\right)+k_{w}\left(1+v^{2}\right)}}\right] .
$$

Die zugehörigen minimalen mittleren Kosten des Wartesystems $M / G / 1$ lauten schließlich

$$
K^{*}=K\left(\lambda^{*}\right)=k_{b}-k_{w}\left(1+v^{2}\right)+\sqrt{k_{w}\left(1+v^{2}\right)\left[2\left(k_{f}-k_{b}\right)+k_{w}\left(1+v^{2}\right)\right]} .
$$


Es ist unmittelbar zu erkennen, daß die kostenminimale Belastung monoton fallend und das Kostenminimum $K^{*}$ monoton steigend bezüglich des Variationskoeffizienten $v$ ist. Die Abb. 2 und 3 veranschaulichen den Verlauf der Funktionen $\lambda^{*}(v)$ und $K^{*}(v)$. Dabei bedeuten die Abkürzungen

$$
\lambda_{0}^{*}=\mu\left[1-\sqrt{\frac{k_{w}}{2\left(k_{f}-k_{b}\right)+k_{w}}}\right]
$$

und

$$
K_{0}^{*}=k_{b}-k_{w}+\sqrt{k_{w}\left[2\left(k_{f}-k_{b}\right)+k_{w}\right]} .
$$

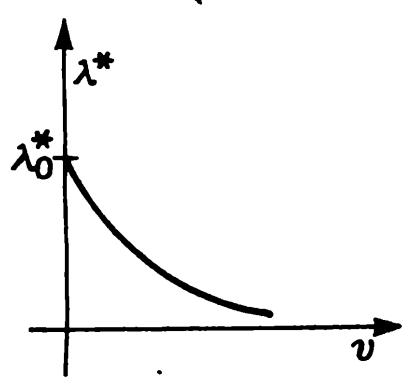

Abb. 2

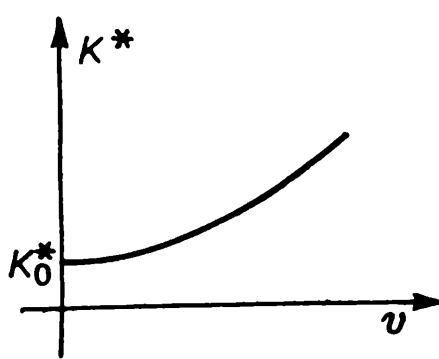

Abb. 3

Demzufolge arbeitet ein einstelliges Wartesystem kostenmäßig um so günstiger, je kleiner die Streuung der Bedienungszeit ist, d. h. je spezialisierter die Bedienungsstelle ist. Ein solches Wartesystem erreicht sein kostengünstigstes Verhalten, wenn es von der Gestalt $M / D / 1$ ist, d. h. wenn die Bedienungszeit konstant ist. In diesem Falle gilt für die kostenminimale Belastung und die minimalen mittleren Kosten $\lambda^{*}=\lambda_{0}^{*}$, $K^{*}=K_{0}^{*}$. Dieses Ergebnis wirft die Frage auf, bis zu welchem Maße die Bedienungsstellen eines mehrstelligen Wartesystems jeweils zu spezialisieren sind, ohne dabei deren Austauschbarkeit vollends aufzugeben. Diese für die Praxis relevante Frage kann in diesem Aufsatz noch nicht beantwortet werden.

\section{Literaturverzeichnis}

[1] M. Fisz, Rachunek prawdopodobieństwa i statystyka matematyczna, PWN, Warszawa 1967.

[2] M. M. Gorbatij, Die Anwendung mathematischer Methoden in der Forschung und Praxis des Seetransportes, Морской флот 24 (1964), S. $12-13$.

[3] H. Krampe, J. Kubát und W. Runge, Bedienungsmodelle, Verlag Dio Wirtschaft, Berlin 1974.

[4] G. Rossa, Zur Optimierung seewärtiger Umschlagssysteme, Seewirtschaft 4 (1972), S. $773-777$. 
[5] W. Runge, Kostenminimale Dimensionierung offener Wartesysteme, Rechentechnik und Datenverarbeitung 10 (1973), Beiheft S. 55-60.

SEKTION SOZIAL. BETRIEBSWIRTSCHAFT

UNIVERSITÄT ROSTOCK

DDR-25 ROSTOCK

Eingegangen am 8. 4. 1974

W. RUNGE (Rostock)

NAJT ANSSZE OBCIAZŹENIE

OTWARTYCH SYSTEMóW OBSEUGI MASOWEJ Z WEJSCIEM POISSONOWSKIM

\section{STRESZCZENIE}

W pracy bada się otwarte systemy obsługi masowej typu $M / M / s$ i $M / G / 8$. W szczególności odpowiada się na pytanie, jaka powinna być intensywność wejść, aby średnie koszty otwartego systemu były minimalne. Podaje się i objaśnia kilka sposobów obliczania takiego obciążenia systemu. 